\title{
Family Physician Participation in Maintenance of Certification
}

Imam M. Xierali, $P b D^{1}$

Jason C. B. Rinaldo, $P b D^{2}$

Larry A. Green, $M D^{2,3}$

Stephen M. Petterson, $\mathrm{PbD}^{1}$

Robert L. Pbillips, Jr, MD, MSPH ${ }^{1}$

Andrew W. Bazemore, MD, MPH

Warren P. Newton, $M D^{2,4}$

James C. Puffer, $M D^{2}$

'The Robert Graham Center for Policy Studies in Family Medicine and Primary Care, Washington, DC

${ }^{2}$ The American Board of Family Medicine, Lexington, Kentucky

${ }^{3}$ Department of Family Medicine, University of Colorado at Denver, Aurora, Colorado

${ }^{4}$ Department of Family Medicine, University of North Carolina at Chapel Hill, Chapel Hill, North Carolina

\begin{abstract}
PURPOSE The American Board of Family Medicine has completed the 7-year transition of all of its diplomates into Maintenance of Certification (MOC). Participation in this voluntary process must be broad-based and balanced for MOC to have any practical national impact on health care. This study explores family physicians' geographic, demographic, and practice characteristics associated with the variations in MOC participation to examine whether MOC has potential as a viable mechanism for dissemination of information or for altering practice.
\end{abstract}

METHODS To investigate characteristics associated with differential participation in MOC by family physicians, we performed a cross-sectional comparison of all active family physicians using descriptive and multinomial logistic regression analyses.

RESULTS Eighty-five percent of active family physicians in this study $(n=70,323)$ have current board certification. Ninety-one percent of all active board-certified family physicians eligible for MOC are participating in MOC. Physicians who work in poorer neighborhoods (odds ratio $[\mathrm{OR}]=1.105 ; 95 \%$ confidence interval [Cl], 1.038-1.176), who are US-born or foreign-born international medical graduates $(\mathrm{OR}=1.444 ; 95 \% \mathrm{Cl}, 1.238-1.684 ; \mathrm{OR}=1.221 ; 95 \% \mathrm{Cl}, 1.124-1.326$, respectively), or who are solo practitioners $(\mathrm{OR}=1.460 ; 95 \% \mathrm{Cl}, 1.345-1.585$ ) are more likely to have missed initial MOC requirements than those from a large, undifferentiated reference group of certified family physicians. When age is held constant, female physicians are less likely to miss initial $\mathrm{MOC}$ requirements $(\mathrm{OR}=0.849 ; 95 \% \mathrm{Cl}, 0.794-0.908)$. Physicians practicing in rural areas were found to be performing similarly in meeting initial $M O C$ requirements to those in urban areas ( $\mathrm{OR}=0.966 ; 95 \% \mathrm{Cl}, 0.919-1.015$, not significant).

CONCLUSION Large numbers of family physicians are participating in MOC. The significant association between practicing in underserved areas and lapsed board certification, however, warrants more research examining causes of differential participation. The penetrance of MOC engagement shows that MOC has the potential to convey substantial practice-relevant medical information to physicians. Thus, it offers a potential channel through which to improve health care knowledge and medical practice.

Ann Fam Med 2009;203-210. doi:10.1370/afm.1251.

\section{INTRODUCTION}

1 aintenance of Certification (MOC) was approved by the American Board of Medical Specialties (ABMS) in 2000 and adopted by ABMS member boards to promote improvement in the quality of care delivered by certified physicians. The move by ABMS member boards from assessors of competency to agents of quality improvement required a transition from encouraging lifelong learning and performing intermittent recertification to more continuous assessment of professionalism, lifelong learning, cognitive expertise, and performance in practice. This new approach is also an effort to increase public accountability by boards and the physicians they certify. Whether these aims are being met requires regular assessment of the $\mathrm{MOC}$ process as it matures, including 
evaluation of physician participation. ${ }^{1}$ If $\mathrm{MOC}$ is to have any impact on health care quality, that effect will be mediated by participation level across geographic boundaries and practice types.

Among the few studies that have addressed the potential barriers to primary care physicians' participation in MOC, Lipner et al and Freed et al found that internal medicine and general pediatric physicians perceived time, expense, and lack of professional necessity as reasons for not participating in MOC., ${ }^{2,3}$ A recent exchange regarding the advantages and disadvantages of participation in MOC in internal medicine echoes these findings. ${ }^{4,5}$ Within family medicine, the same sentiments have been expressed. ${ }^{6-9}$ Very few studies to date have examined differential participation in MOC.

The American Board of Family Medicine (ABFM) began the transition of all diplomates into its MOC process, the Maintenance of Certification for Family Physicians (MC-FP), in 2003. Having completed this transition in 2010, the ABFM is now uniquely situated to answer critical questions regarding the adoption of this new paradigm by all family physicians. In an effort to understand and describe the impact of the transition to MC-FP, the geographic distribution of this impact, and the related practice demographics, Bazemore et al undertook an analysis of the participation of a single cohort of family physicians participating in MC-FP. ${ }^{10}$ This study showed wide penetration of MC-FP across the United States with relatively uniform participation rates in rural, suburban, and urban locales. Although this lack of geographic variation is reassuring, lower uptake of MC-FP among physicians serving underserved populations motivated further exploration of the differential impact of MC-FP requirements on family physicians. In this study, a more intensive investigation was undertaken to explore the geographic, demographic, and practice characteristics associated with the variations in board certification and MC-FP participation.

\section{METHODS}

\section{Study Sample}

Study subjects included all active physicians recertifying, initially certifying, or attempting certification after prior failure with the ABFM. The ABFM administrative records and the 2009 American Medical Association (AMA) Physician Masterfile were linked and overlaid upon various geographies, including census 2000 tabulation, the federally designated Health Professional Shortage Areas (HPSAs), Medically Underserved Areas/Populations (MUA/P), and Primary Care Service Areas (PCSAs). Cohorts were developed based on certification status and MC-FP participation. Relationships between family physicians' participation level in ABFM certification and physician characteristics and geographic distribution were then analyzed.

ABFM administrative data provides addresses, demographic characteristics, practice characteristics, and test outcomes of those who have ever attempted, obtained, or renewed certification. With complementary demographic data pertaining to physician specialties, practice type, employment setting, and primary profession from the AMA Masterfile and spatial data joining, a rich analytical data set was constructed that includes year of birth, sex, address, physician practice type, employment type (eg, group, independent/solo, partnership, federal, military), and primary profession. Those who certified or recertified in 2009 entered MC-FP in 2010 and thus were not yet in MC-FP in 2009; they were therefore dropped from the analysis. Those who were residents, retired, semiretired, or unclassified are regarded as inactive and also were excluded from further analysis. Active physicians were defined as those physicians in direct patient care, doing research, teaching, or in administrative functions who were still active in their employment.

\section{Outcome Measures}

The MC-FP paradigm provides family physicians meeting requirements in a timely manner with 10 -year certification rather than the traditional 7-year period. Those who finish a combination of 3 self-assessment modules (SAMs) or Performance in Practice Modules (PPMs) within the first 3 years after their examination (termed Stage I) and fulfill these requirements again in the next 3 years (Stage II) will have their certification extended to 10 years. Those who do not complete the 2, 3-year stages will remain in a 7-year recertification cycle, but they must complete 6 SAMs and 1 PPM to be eligible for the next recertification examination.

For analyses, family physicians were divided into 1 of 6 mutually exclusive categories, based on their current certification status and involvement in MC-FP processes:

1. Certified: Stage I requirements completed (eligible for 10-year certification)

2. Certified: Stage I active (still eligible for 10-year certification, the Stage I window is still open but requirements are not yet complete)

3. Certified: Stage I incomplete (7-year certification; Stage I window is closed and fewer than 3 MC-FP activities were completed)

4. Not certified: certification lapsed without further examination attempts

5. Not certified: certification lapsed, failed last examination 
6. Not certified: certification revoked (lost medical license, disciplinary action, or restricted license)

\section{Statistical Analyses}

Analyses include $\chi^{2}$ tests and generalized multinomial logistic regression, focusing on MC-FP certification status as described above. A stepwise selection method was then used to build a parsimonious regression model from the physician characteristics. An interaction term between age and sex was added into the model because of the observation that female physicians tend to be younger. Probabilities lower than 5\% were deemed significant.

\section{RESULTS}

We matched 91,272 (91\%) of ABFM records in the AMA Masterfile. No AMA match was found for 9,053 ABFM physicians (Figure 1). Among the unmatched physicians, $15 \%$ were deceased, $67 \%$ were older than 65 years, and $5 \%$ were younger than 35 years as of 2009 .

Those physicians $(11,968)$ who were deceased, retired, or aged 75 years and older were filtered from subsequent analyses. Within the matched and filtered cases, 10,444 (13\%) of 79,304 physicians had lapsed certification, most of whom made no further attempts to recertify $(7,825)$. Those physicians who certified or recertified in 2009 entered MC-FP in 2010 and thus were dropped from MC-FP participation analysis $(8,981)$. It is worth noting that there were 149 family physicians aged 75 years and older actively participating in the new MC-FP process.

Participation rates in both board certification and MC-FP were high (Table 1). Eighty-five percent of all active family physicians $(70,323$ with matched data in this study) were currently board certified, and $91 \%$ of all active board-certified family physicians eligible for MC-FP $(59,879)$ were participating in MC-FP.

\section{Association Analysis}

Using $\chi^{2}$ analyses, we found that across each of the physician age categories, increasing age is significantly associated with decreasing likelihood to engage in MC-FP (Table 2). Ninety percent of female family physicians were participating in MC-FP compared with $83 \%$ of male physicians. Female physicians were also much less likely to have lapsed certification than were male physicians.

\section{Table 1. Participation Level Into MC-FP by Each} Group of Active Diplomates

\begin{tabular}{lcc}
\hline & $\begin{array}{c}\text { At least 1 } \\
\text { SAM or PPM } \\
\text { MC-FP Participation Status }\end{array}$ & $\begin{array}{c}\text { Group } \\
\text { Total }\end{array}$ \\
\hline Administrative or license issue & $39(0.13)$ & 235 \\
Chose to lapse & - & 7,825 \\
Failed examination & - & 2,384 \\
MC-FP, 7-year pathway & $1,027(3.36)$ & 6,272 \\
Stage I & $4,443(14.54)$ & 28,551 \\
Stage I complete & $25,056(81.98)$ & 25,056 \\
Total & 30,565 (100) & 70,323 \\
\hline MC-FP = Maintenance of Certification for Family Physicians; PPM = Perfor- \\
mance in Practice Module; SAM = Self-Assessment Module. \\
Note: Participation in MC-FP is evidenced by taking 1 or more SAM or PPM. \\
\hline
\end{tabular}

There are significant differences within the internationally trained family medicine community. US-born international medical graduates (IMGs) were slightly less likely to be on track for MC-FP than were foreignborn IMGs and were more likely to have lapsed or not achieved certification (Table 1). The foreign-born IMG group was more similar in MC-FP participation to medical graduates from the United States and Canada (non-IMG) than were the US-born IMGs.

Practice type and setting were also significantly associated with important differences in MC-FP participation. Physicians in direct patient care, either office- 
Table 2. Associations Between Practice Type, International Medical Graduate Status, Employment Type, With MC-FP Status ( $=70,323$ Physicians)

\begin{tabular}{|c|c|c|c|c|c|c|}
\hline \multirow[b]{2}{*}{ Variable } & \multicolumn{3}{|c|}{ Not Certified ${ }^{a}$} & \multicolumn{3}{|c|}{ Currently Certified ${ }^{b}$} \\
\hline & $\begin{array}{c}\text { Administrative } \\
\text { or License Issue } \\
\text { n (\%) }\end{array}$ & $\begin{array}{l}\text { Chose } \\
\text { to Lapse } \\
\text { n (\%) }\end{array}$ & $\begin{array}{c}\text { Failed } \\
\text { Examination } \\
\text { n (\%) }\end{array}$ & $\begin{array}{l}\text { MC-FP } \\
\text { 7-Yearb } \\
\text { n (\%) }\end{array}$ & $\begin{array}{c}\text { Stage } \mathbf{I}^{\mathbf{b}} \\
\mathbf{n}(\%)\end{array}$ & $\begin{array}{c}\text { Stage I } \\
\text { Complete and } \\
\text { Beyond } \mathbf{d}^{\mathrm{b}} \\
\mathrm{n}(\%)\end{array}$ \\
\hline Physicians & $235(0.33)$ & 7,825 (11.13) & $2,384(3.39)$ & $6,272(8.92)$ & $28,551(40.60)$ & $25,056(35.63)$ \\
\hline At least 1 MC-FP activity,d & $39(0.13)$ & $0(0)$ & $0(0)$ & $1,027(3.36)$ & $4,443(14.54)$ & $25,056(81.98)$ \\
\hline \multicolumn{7}{|l|}{ Age, $y^{d}$} \\
\hline$<35$ & $2(0.04)$ & $1(0.02)$ & $1(0.02)$ & $256(4.84)$ & $3,187(60.23)$ & $1,844(34.85)$ \\
\hline $35<45$ & $45(0.21)$ & $652(3.06)$ & $297(1.39)$ & $1,751(8.22)$ & $9,772(45.88)$ & $8,781(41.23)$ \\
\hline $45<55$ & $94(0.4)$ & $2,179(9.36)$ & 720 (3.09) & $2,254(9.68)$ & $9,299(39.94)$ & $8,735(37.52)$ \\
\hline $55<65$ & $78(0.46)$ & $3,456(20.3)$ & $861(5.06)$ & $1,681(9.87)$ & $5,741(33.72)$ & $5,209(30.59)$ \\
\hline $65<75$ & $16(0.47)$ & $1,537(44.98)$ & $505(14.78)$ & $329(9.63)$ & $552(16.15)$ & 478 (13.99) \\
\hline \multicolumn{7}{|l|}{$\operatorname{Sex}^{d}$} \\
\hline Male & $207(0.44)$ & $6,168(13.24)$ & $1,732(3.72)$ & $4,548(9.76)$ & $18,302(39.29)$ & $15,628(33.55)$ \\
\hline Female & $28(0.12)$ & $1,657(6.98)$ & $652(2.75)$ & $1,724(7.26)$ & $10,249(43.18)$ & $9,428(39.72)$ \\
\hline \multicolumn{7}{|l|}{ Training ${ }^{d}$} \\
\hline Non-IMG & $199(0.34)$ & $6,712(11.33)$ & $1,436(2.42)$ & $5,170(8.72)$ & $24,423(41.22)$ & $21,317(35.97)$ \\
\hline Foreign-born IMG & $23(0.26)$ & $790(8.8)$ & $722(8.04)$ & $858(9.56)$ & $3,476(38.73)$ & $3,106(34.61)$ \\
\hline US-born IMG & $13(0.62)$ & $323(15.45)$ & $226(10.81)$ & $244(11.67)$ & $652(31.18)$ & $633(30.27)$ \\
\hline \multicolumn{7}{|l|}{ Practice type ${ }^{d}$} \\
\hline Direct patient care & $224(0.33)$ & $7,300(10.86)$ & $2,264(3.37)$ & $6,044(8.99)$ & $27,410(40.78)$ & $23,971(35.66)$ \\
\hline Administration & $2(0.15)$ & $303(22.13)$ & $77(5.62)$ & $108(7.89)$ & $461(33.67)$ & $418(30.53)$ \\
\hline Medical teaching & $3(0.23)$ & $104(7.85)$ & $19(1.44)$ & $91(6.87)$ & $567(42.82)$ & $540(40.79)$ \\
\hline Medical research & $2(0.96)$ & $60(28.71)$ & $11(5.26)$ & $15(7.18)$ & $60(28.71)$ & 61 (29.19) \\
\hline Nonpatient care & $4(1.92)$ & $58(27.88)$ & $13(6.25)$ & $14(6.73)$ & $53(25.48)$ & $66(31.73)$ \\
\hline \multicolumn{7}{|l|}{ Employment setting ${ }^{d}$} \\
\hline Solo practice & $55(0.58)$ & $1,911(20.07)$ & $739(7.76)$ & $1,037(10.89)$ & $2,974(31.23)$ & $2,808(29.48)$ \\
\hline Group practice & $98(0.25)$ & $3,221(8.34)$ & $934(2.42)$ & $3,335(8.63)$ & $16,179(41.87)$ & 14,875 (38.49) \\
\hline Institution & $22(0.31)$ & $1,065(14.81)$ & $233(3.24)$ & $578(8.04)$ & $2,713(37.74)$ & $2,578(35.86)$ \\
\hline Federal & $1(0.25)$ & $53(13.05)$ & $17(4.19)$ & $43(10.59)$ & $141(34.73)$ & 151 (37.19) \\
\hline Military & $9(0.42)$ & $196(9.07)$ & $76(3.52)$ & $187(8.65)$ & $837(38.71)$ & 857 (39.64) \\
\hline Local, state & $3(0.26)$ & $251(21.85)$ & $60(5.22)$ & $92(8.01)$ & $361(31.42)$ & $382(33.25)$ \\
\hline Government agencies & $47(0.42)$ & $1,128(10.03)$ & $325(2.89)$ & $1,000(8.89)$ & $5,346(47.52)$ & $3,405(30.26)$ \\
\hline Others & $55(0.58)$ & $1,911(20.07)$ & $739(7.76)$ & 1,037 (10.89) & $2,974(31.23)$ & $2,808(29.48)$ \\
\hline \multicolumn{7}{|l|}{ Major professional activities ${ }^{d}$} \\
\hline Administration & $2(0.15)$ & $303(22.13)$ & $77(5.62)$ & $108(7.89)$ & $461(33.67)$ & $418(30.53)$ \\
\hline Hospital full-time & $18(0.29)$ & $781(12.42)$ & $209(3.32)$ & $563(8.95)$ & $2,410(38.33)$ & 2,307 (36.69) \\
\hline Medical teaching & $3(0.23)$ & $104(7.85)$ & $19(1.44)$ & $91(6.87)$ & $567(42.82)$ & $540(40.79)$ \\
\hline Office-based & $204(0.34)$ & $6,490(10.69)$ & $2,041(3.36)$ & $5,461(8.99)$ & $24,938(41.07)$ & $21,585(35.55)$ \\
\hline Research & $2(0.96)$ & $60(28.71)$ & $11(5.26)$ & $15(7.18)$ & $60(28.71)$ & $61(29.19)$ \\
\hline Others & $6(1.45)$ & $87(21.07)$ & $26(6.30)$ & $34(8.23)$ & $115(27.85)$ & $145(35.11)$ \\
\hline
\end{tabular}

based or hospital-based, and medical teaching were more likely to be engaged in MC-FP than were those in medical research, administrative, and other nonpatient care roles. Family physicians in group practice were more than twice as likely to be board certified and were $10 \%$ more likely to be engaged in MC-FP than were solo family physicians. Those employed by the military were much more likely to be certified and involved in MC-FP, whereas those employed by local government health agencies were not.
Physician status in MC-FP also varied along geographic dimensions. Through address geocoding, 99\% of physicians were linked to census geographies and federally designated shortage areas. A small but statistically significant difference was noted in lapsed certification for physicians in HPSAs (16\%), MUA/ Ps $(17 \%)$ and poorer neighborhoods (16\%) than in non-HPSAs (14\%), non-MUA/Ps (14\%), and wealthier neighborhoods (13\%). Rural physicians were slightly more likely than their urban counterparts to maintain 


\begin{tabular}{|c|c|c|c|c|c|c|}
\hline \multirow[b]{2}{*}{ Variable } & \multicolumn{3}{|c|}{ Not Certified $^{a}$} & \multicolumn{3}{|c|}{ Currently Certified $^{\mathrm{b}}$} \\
\hline & $\begin{array}{l}\text { Administrative } \\
\text { or License Issue } \\
\quad \mathrm{n}(\%)\end{array}$ & $\begin{array}{l}\text { Chose } \\
\text { to Lapse } \\
\text { n (\%) }\end{array}$ & $\begin{array}{l}\text { Failed } \\
\text { Examination } \\
n(\%)\end{array}$ & $\begin{array}{l}\text { MC-FP } \\
\text { 7-Yearb } \\
\text { n (\%) }\end{array}$ & $\begin{array}{c}\text { Stage } \mathrm{I}^{\mathrm{b}} \\
\mathrm{n}(\%)\end{array}$ & $\begin{array}{c}\text { Stage I } \\
\text { Complete and } \\
\text { Beyond }{ }^{\mathrm{b}} \\
\text { n (\%) }\end{array}$ \\
\hline \multicolumn{7}{|l|}{ Geography } \\
\hline HPSA $^{d}$ & $77(0.45)$ & $2,059(12)$ & $615(3.58)$ & $1,621(9.45)$ & $6,949(40.5)$ & $5,839(34.03)$ \\
\hline Non-HPSA & $157(0.30)$ & $5,594(10.59)$ & $1,752(3.32)$ & $4,625(8.76)$ & $21,534(40.78)$ & $19,148(36.26)$ \\
\hline $\mathrm{MUA} / \mathrm{P}^{\mathrm{d}}$ & $63(0.41)$ & $1,980(12.73)$ & 617 (3.97) & $1,496(9.62)$ & 6,181 (39.74) & $5,218(33.55)$ \\
\hline Non-MUA/P & $171(0.31)$ & $5,673(10.43)$ & $1,750(3.22)$ & $4,750(8.73)$ & $22,302(40.99)$ & $19,769(36.33)$ \\
\hline Rurald $^{d}$ & $52(0.4)$ & $1,481(11.42)$ & $335(2.58)$ & $1,219(9.4)$ & $5,423(41.83)$ & 4,453 (34.35) \\
\hline Urban & $182(0.32)$ & $6,088(10.72)$ & $2,007(3.53)$ & $5,001(8.80)$ & $23,029(40.54)$ & $20,496(36.08)$ \\
\hline $\begin{array}{l}\text { Area } \geq 20 \% \text { under } 200 \% \\
\quad \text { federal poverty level }\end{array}$ & $131(0.38)$ & $4,215(12.32)$ & $1,190(3.48)$ & $3,277(9.58)$ & $13,834(40.42)$ & $11,577(33.83)$ \\
\hline $\begin{array}{l}\text { Area }<20 \% \text { under } 200 \% \\
\quad \text { federal poverty level }\end{array}$ & $103(0.29)$ & $3,438(9.62)$ & $1,177(3.29)$ & $2,968(8.30)$ & $14,648(40.98)$ & 13,407 (37.51) \\
\hline $\begin{array}{l}\text { PCSA population to physi- } \\
\text { cian ratio }<1500: 1^{d}\end{array}$ & $139(0.32)$ & $4,509(10.41)$ & $1,327(3.06)$ & $3,692(8.53)$ & 17,796 (41.09) & $15,843(36.58)$ \\
\hline $\begin{array}{l}\text { PCSA population to phy- } \\
\text { sician ratio }>1500: 1\end{array}$ & $93(0.36)$ & $2,962(11.55)$ & $994(3.88)$ & $2,458(9.59)$ & $10,315(40.23)$ & 8,818 (34.39) \\
\hline \multicolumn{7}{|c|}{$\begin{array}{l}\text { IMG = international medical graduate; HPSA = Health Professional Shortage Area; MC-FP = Maintenance of Certification for Family Physicians; MUA } / P=\text { Medically } \\
\text { Underserved Area/Population; non-IMG = graduates from US or Canadian medical schools; PCSA = Primary Care Service Area; PPM = Performance in Practice Module; } \\
\text { SAM = Self-Assessment Module. }\end{array}$} \\
\hline $\begin{array}{l}\text { a Certificate expired as of the begir } \\
\text { b Certificate current as of the begin } \\
\text { ' Completing at least } 1 \text { SAM or PPI } \\
\text { d Fequencies differ significantly at }\end{array}$ & $\begin{array}{l}\text { ing of } 2009 . \\
\text { ng of } 2009 . \\
\text { module. } \\
<.0001 .\end{array}$ & & & & & \\
\hline
\end{tabular}

their certification ( $86 \%$ vs $85 \%$ ). Physicians practicing in a PCSA with a population-to-physician ratio of less than 1500 to 1 (better than average supply of primary care physicians) also tended to maintain their board certification better than those outside such areas $(86 \%$ vs $84 \%)$.

\section{Multinomial Logistic Regression Analysis}

To determine whether any of the observed associations held when controlling for the remaining variables, multinomial logistic regression was used to predict membership in the 6 participation categories. The largest and most generic category, Stage I active (certified, started MC-FP), was selected as the reference group, because certified physicians who have not yet differentiated into 7- or 10-year MC-FP pathways provide a useful benchmark to compare with the other 5 participation categories.

When accounting for conceptually similar variables, such as MUA/P, percentage of population in poverty, and other geographic variables, HPSA was no longer significantly associated with MOC. The interaction between age and sex showed a significant effect when discerning MC-FP 7-year pathway physicians from those still in Stage I. The logistic regression generated acceptable overall model fit, and results of the likelihood ratio test and efficient score test reject the hypothesis that all slope parameters are equal to zero.
The regression outputs suggest interesting patterns in physicians' participation level in the MC-FP processes (Table 3). Holding other variables constant, older physicians were more likely to have lapsed certification. When holding age constant, however, female physicians were less likely to have a revoked certification because of an administrative or license issue, less likely to have chosen the MC-FP 7-year pathway, and more likely to have completed Stage I. Foreign-born IMGs were less likely to have allowed their certification to lapse but more likely to have failed examinations. They were also more likely to have not met their initial MC-FP requirements and thus defaulted into the 7-year pathway. Moreover, the US-born IMGs were more likely to have failed their examinations and to have also not met their initial MC-FP requirements and thus defaulted into the 7-year pathway when compared with the reference group. Physicians employed in city, county, and state health agencies and institutions were significantly more likely than the reference group to have let their certification lapse by choice. Solo practitioners were more likely to have had revoked certification owing to administrative or licensure issues, let their certification lapse, or have failed their examination, and more likely to have chosen the 7-year pathway compared with group practitioners. Compared with office-based physicians, teaching physicians were less likely to have allowed their certificate 
Table 3. Odds Ratio Estimates of Multinomial Logistic Regression of Active Physician's MC-FP Status

\begin{tabular}{|c|c|c|c|c|c|c|c|c|c|c|}
\hline \multirow[b]{2}{*}{ Parameters } & \multicolumn{2}{|c|}{$\begin{array}{l}\text { Administrative } \\
\text { or License Issue } \\
(n=232)\end{array}$} & \multicolumn{2}{|c|}{$\begin{array}{l}\text { Chose to Lapse } \\
\qquad(\mathrm{n}=7,471)\end{array}$} & \multicolumn{2}{|c|}{$\begin{array}{c}\text { Failed } \\
\text { Examination } \\
(n=2,321)\end{array}$} & \multicolumn{2}{|c|}{$\begin{array}{c}\text { MC-FP 7-Year } \\
\text { Pathway } \\
(n=6,149)\end{array}$} & \multicolumn{2}{|c|}{$\begin{array}{c}\text { Stage I } \\
\text { Complete } \\
(n=24,658)\end{array}$} \\
\hline & OR & $95 \% \mathrm{Cl}$ & OR & $95 \% \mathrm{Cl}$ & OR & $95 \% \mathrm{Cl}$ & OR & $95 \% \mathrm{Cl}$ & OR & $95 \% \mathrm{Cl}$ \\
\hline $\begin{array}{l}\text { Age at sex }= \\
\text { female }\end{array}$ & 1.093 & $1.049-1.140$ & 1.139 & 1.131-1.146 & 1.118 & 1.107-1.128 & 1.000 & $1.000-1.000$ & 1.000 & $1.000-1.000$ \\
\hline Age at sex $=$ male & 1.053 & $1.036-1.070$ & 1.141 & 1.137-1.146 & 1.120 & 1.113-1.127 & 1.034 & $1.030-1.037$ & 1.000 & $1.000-1.000$ \\
\hline $\begin{array}{c}\text { Female vs male at } \\
\text { age }=43.459\end{array}$ & 0.255 & $0.154-0.421$ & 0.907 & $0.822-1.001$ & 1.113 & $0.958-1.294$ & 0.849 & $0.794-0.908$ & 1.098 & $1.059-1.138$ \\
\hline $\begin{array}{l}\text { Foreign-born IMG } \\
\text { vs non-IMG }\end{array}$ & 0.836 & $0.538-1.298$ & 0.768 & $0.701-0.842$ & 3.255 & $2.931-3.615$ & 1.221 & $1.124-1.326$ & 1.050 & 0.995-1.107 \\
\hline $\begin{array}{l}\text { US-born IMG vs } \\
\text { non-IMG }\end{array}$ & 1.660 & $0.937-2.943$ & 1.047 & $0.902-1.216$ & 3.436 & $2.892-4.083$ & 1.444 & $1.238-1.684$ & 1.122 & $1.003-1.255$ \\
\hline \multicolumn{11}{|c|}{ Employment (reference $=$ group practice) } \\
\hline City, county, state & 1.079 & $0.310-3.751$ & 2.067 & $1.695-2.522$ & 1.584 & $1.149-2.184$ & 1.154 & $0.899-1.482$ & 1.125 & $0.964-1.314$ \\
\hline Federal & 1.539 & $0.182-13.030$ & 1.234 & $0.847-1.798$ & 1.401 & $0.780-2.513$ & 1.236 & $0.836-1.827$ & 1.226 & $0.949-1.585$ \\
\hline Institution & 1.523 & $0.749-3.099$ & 1.526 & $1.328-1.753$ & 1.092 & $0.857-1.393$ & 0.877 & $0.741-1.038$ & 1.053 & $0.957-1.159$ \\
\hline Military & 2.896 & $0.975-8.603$ & 1.088 & $0.862-1.372$ & 1.215 & $0.833-1.773$ & 0.916 & $0.714-1.176$ & 1.176 & $1.016-1.362$ \\
\hline Solo practice & 2.251 & $1.599-3.170$ & 2.064 & $1.914-2.225$ & 2.405 & $2.153-2.688$ & 1.460 & $1.345-1.585$ & 1.041 & $0.983-1.102$ \\
\hline Others & 2.388 & $1.651-3.452$ & 2.364 & $2.168-2.577$ & 1.687 & $1.464-1.943$ & 1.049 & $0.968-1.137$ & 0.677 & $0.645-0.712$ \\
\hline \multicolumn{11}{|c|}{ Primary professional activities (reference $=$ office-based) } \\
\hline Administration & 0.156 & $0.020-1.189$ & 1.008 & 0.841-1.209 & 1.301 & 0.971-1.743 & 0.937 & $0.740-1.187$ & 0.937 & $0.808-1.087$ \\
\hline Hospital full-time & 0.614 & $0.249-1.517$ & 1.090 & $0.923-1.288$ & 1.115 & $0.834-1.490$ & 1.204 & $0.988-1.467$ & 0.956 & $0.851-1.073$ \\
\hline Medical teaching & 0.472 & $0.137-1.627$ & 0.392 & $0.308-0.501$ & 0.371 & $0.226-0.611$ & 0.713 & $0.557-0.913$ & 1.012 & $0.886-1.156$ \\
\hline Research & 2.982 & $0.657-13.537$ & 2.127 & $1.423-3.180$ & 2.025 & 1.027-3.991 & 1.172 & $0.656-2.094$ & 1.049 & $0.728-1.510$ \\
\hline Others & 5.873 & $2.158-15.981$ & 1.800 & $1.305-2.484$ & 2.413 & $1.495-3.893$ & 1.468 & $0.978-2.205$ & 1.271 & $0.982-1.647$ \\
\hline \multicolumn{11}{|l|}{ Geography } \\
\hline Rural & 0.989 & $0.699-1.400$ & 0.826 & $0.765-0.893$ & 0.667 & $0.582-0.764$ & 0.910 & $0.841-0.984$ & 0.966 & $0.919-1.015$ \\
\hline MUA/P & 1.169 & $0.850-1.607$ & 1.111 & $1.035-1.191$ & 1.322 & $1.182-1.477$ & 1.062 & $0.989-1.141$ & 0.999 & $0.955-1.046$ \\
\hline $\begin{array}{l}\text { Has } 20 \% \text { or } \\
\text { more under } \\
200 \% \text { poverty }\end{array}$ & 1.156 & $0.861-1.551$ & 1.100 & $1.034-1.171$ & 0.971 & 0.879-1.072 & 1.105 & 1.038-1.176 & 0.934 & $0.898-0.971$ \\
\hline $\begin{array}{l}\text { PCSA population } \\
\text { to physician } \\
\text { ratio <1500:1 }\end{array}$ & 1.012 & $0.769-1.333$ & 0.953 & 0.898-1.010 & 0.817 & $0.744-0.896$ & 0.921 & $0.868-0.977$ & 1.018 & $0.981-1.057$ \\
\hline
\end{tabular}

to lapse, less likely to have failed an examination, and less likely to have taken the 7-year pathway. Those physicians whose primary profession was research, however, were more likely than office-based physicians to have allowed their certification to lapse or to have failed their examinations.

Physicians practicing in rural areas were less likely to have let their certification lapse and less likely to have chosen the 7-year pathway than to be engaged in Stage I. Physicians in the medically underserved areas, however, were more likely to have let their board certification lapse by choice or because they failed their examinations. Physicians in poorer neighborhoods also were more likely to have allowed their board certification to lapse and more likely to have chosen the 7-year pathway than to be engaged in Stage I. They were also less likely to have completed Stage I.

\section{DISCUSSION}

This study examined physician characteristics related to MC-FP participation rates and to board certification. The debate about whether to participate in MOC (generically) appears to be less essential to family physicians than the choice of a particular participation route (selecting a 7 - or a 10 -year recertification cycle).

Insofar as physician participation must fully mediate any potential benefit of MC-FP, lower participation limits this potential value. The core finding of this study is the widespread participation in MC-FP. The penetrance of MC-FP engagement shows that it has the potential to convey substantial practice-relevant medical information to physicians. Thus, it has potential as a delivery tool to address the attrition of knowledge and skills that can occur across a medical career. ${ }^{11}$ 
Evaluating whether this potential is realized will take time and careful consideration.

Despite that the ABFM certification has always been voluntary, time-limited, and requiring recertification, well more than 100,000 family physicians have engaged in the certification process to date. The great majority of family physicians in the United States maintains board certification and is actively engaged in the MC-FP process. For the substantial number of active physicians who have not maintained certification or engaged in the MC-FP process, this study reveals some important patterns.

The study found that family physicians practicing in poorer and underserved areas were more likely to have allowed board certification to lapse. Lower participation in the certification process might be a local phenomenon related to an inadequate supply of physicians in these areas. Employers may simply not require evidence of certification as a condition for employment. Several recent studies have found a positive association between board certification and the quality of clinical care. ${ }^{12-16}$ Physicians demonstrably less committed to MC-FP may therefore compound the problem of low supply in underserved areas with additional medical knowledge attrition. Other strong possibilities to explain why physicians in poorer and underserved areas let their certification lapse include higher time demands and fewer resources to support MC-FP efforts. These possibilities are consistent with the characteristics of poor and underserved areas, as these areas generally have limited health care providers and technological resources.

The interaction between age and physician sex may be explained by the increased entry of women into family medicine in more recent years and the greater likelihood of older physicians to have allowed their certification to lapse. When controlling for age however, women are still significantly more likely than men to have completed the first stage of $\mathrm{MC}-\mathrm{FP}_{i}$ men are significantly more likely to lose certification because of administrative or licensure issues.

Several limitations in this study should be noted. Although all family physicians have transitioned into MC-FP, not all have had sufficient time to declare their MC-FP pathways. The observed participation pattern might change when all family physicians have selected their preferred participation pathway by the end of 2016. A considerable time lag in physician retirement records may be reflected in the AMA Masterfile, which may have resulted in the inclusion of some inactive physicians in our analysis. The large sample size of the study allows detection of statistically significant but practically small differences. The generalizability of these findings to other ABMS boards may be limited.
The specialty of family medicine may involve incentive structures and individual motivators that are different from those of other specialties, especially those outside primary care. Finally, increasing practice-relevant knowledge through MC-FP may improve quality, but until more compelling data are forthcoming, mere participation in MC-FP may be at best an imperfect indicator of quality at the present time. Nevertheless, this study provides new insights into trends in family physician participation in MC-FP.

In conclusion, participation in MC-FP appears to be robust. Large numbers of family physicians are embracing MC-FP and meeting its requirements in a timely fashion. Even so, a substantial number of active physicians have not participated in MC-FP and have allowed their certification to lapse. As more studies have linked quality of medical care to board certification, ${ }^{17,18}$ it is particularly troubling that physicians who have not maintained certification tend to be practicing in underserved areas or caring for underserved populations. High levels of health care disparities and the need for high-quality care in these areas make it even more pressing to explore and understand the barriers to participation in maintenance of board certification by these physicians. The causal relationship between practicing in underserved areas and having allowed board certification to lapse is not established in this analysis; nevertheless, their significant association suggests a need for further investigation, policy development, and intervention. Perhaps more importantly, the relation between employment type and likelihood to have let certification lapse indicates policy actions may be needed to support physicians in certain employment settings, in particular those in underserved and impoverished areas. Finally, policies targeted toward US-born family physicians who were trained abroad may be worth considering. Outreach programs may be needed to help US-born IMGs to assess their knowledge base, to keep updated, with latest developments in medicine, and to ensure highquality care to patients.

To read or post commentaries in response to this article, see it online at http://www.annfammed.org/cgi/content/full/9/3/203.

Key words: Certification; education, continuing; maintenance of certification; health care delivery; health services research; quality of health care; health care disparities; health policy research

Submitted August 24, 2010; submitted, revised, December 31, 2010; accepted February 25, 2011.

Acknowledgements: This work was supported by funding from the American Board of Family Medicine Foundation. The authors thank Michael Hagen, MD, for his valuable comments and Martey Dodoo, $\mathrm{PhD}$, for his careful review of the manuscript. We also acknowledge the anonymous reviewers for their improvements to the article. 


\section{References}

1. American Board of Medical Specialties (ABMS). About ABMS/ABMS History [Web page]. http://www.abms.org/About_ABMS/ABMS_History/Extended_History/Competency_Movement.aspx. Accessed Dec 17, 2010.

2. Lipner RS, Bylsma WH, Arnold GK, Fortna GS, Tooker J, Cassel CK. Who is maintaining certification in internal medicine-and why? A national survey 10 years after initial certification. Ann Intern Med. 2006;144(1):29-36.

3. Freed GL, Dunham KM, Althouse LA; American Board of Pediatrics, Research Advisory Committee. Characteristics of general and subspecialty pediatricians who choose not to recertify. Pediatrics. 2008;121(4):711-717.

4. Drazen JM, Weinstein DF. Considering recertification. N Engl J Med. 2010;362(10):946-947.

5. Levinson W, King TE Jr, Goldman L, Goroll AH, Kessler B. Clinical decisions. American Board of Internal Medicine maintenance of certification program. N Engl J Med. 2010;362(10):948-952.

6. Pugh M; American Board of Family Practice. Refining the paradigm: the transition from recertification to maintenance of certification. Ann Fam Med. 2003;1(1):56-58.

7. White B. Are you ready for maintenance of certification? Fam Pract Manag. 2005;12(1):24-48.

8. Loxterkamp D. Five easy answers: where the ABFM cognitive exam has gone wrong. [Essay/Commentary]. Fam Med. 2009; 41(3):210-212.

9. Welk S. MOC triggers resignation. [Letter]. Fam Pract Manag. 2005; 12(3):14.

10. Bazemore AW, Xierali IM, Petterson SM, et al. American Board of Family Medicine (ABFM) maintenance of certification: variations in self-assessment modules uptake within the 2006 cohort. J Am Board Fam Med. 2010;23(1):49-58.
11. Choudhry NK, Fletcher RH, Soumerai SB. Systematic review: the relationship between clinical experience and quality of health care. Ann Intern Med. 2005;142(4):260-273.

12. Ramser PG, Carline JD, Inui TS, Larson EB, Logerfo JP, Weinrich MD. Predictive validity of certification by the American Board of Internal Medicine. Ann Intern Med. 1989;110:719-726.

13. Norcini JJ, Lipner RS, Kimball HR. Certifying examination performance and patient outcomes following acute myocardial infarction. Med Educ. 2002;36(9):853-859.

14. Chen J, Rathore SS, Wang Y, Radford MJ, Krumholz HM. Physician board certification and the care and outcomes of elderly patients with acute myocardial infarction. J Gen Intern Med. 2006;21(3): 238-244.

15. Holmboe ES, Wang Y, Meehan TP, et al. Association between maintenance of certification examination scores and quality of care for medicare beneficiaries. Arch Intern Med. 2008;168(13):1396-1403.

16. Turchin A, Shubina M, Chodos AH, Einbinder JS, Pendergrass ML. Effect of board certification on antihypertensive treatment intensification in patients with diabetes mellitus. Circulation. 2008;117(5): 623-628.

17. Brennan TA, Horwitz RI, Duffy FD, Cassel CK, Goode LD, Lipner RS. The role of physician specialty board certification status in the quality movement. JAMA. 2004;292(9):1038-1043.

18. Norcini JJ, Boulet JR, Dauphinee WD, Opalek A, Krantz ID, Anderson ST. Evaluating the quality of care provided by graduates of international medical schools. Health Aff (Millwood). 2010;29(8): 1461-1468. 